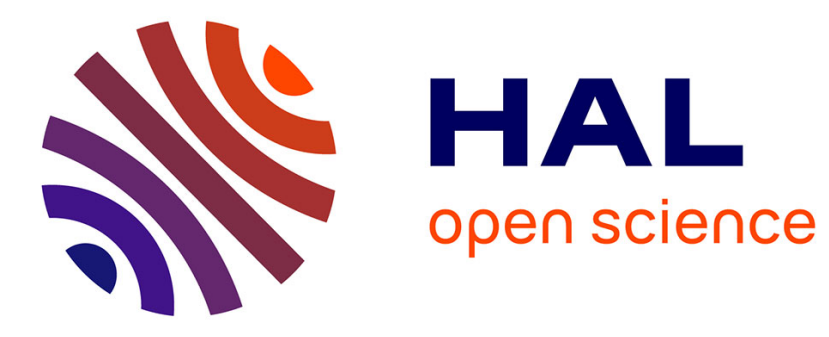

\title{
Post-test perceptions of digital tools by the elderly in an ambient environment
}

Elizabeth Bougeois, Jenny Duchier, Frédéric Vella, Mathilde Blanc Machado, Adrien van den Bossche, Thierry Val, Damien Brulin, Nadine Vigouroux, Eric

Campo

\section{To cite this version:}

Elizabeth Bougeois, Jenny Duchier, Frédéric Vella, Mathilde Blanc Machado, Adrien van den Bossche, et al.. Post-test perceptions of digital tools by the elderly in an ambient environment. 15th International Conference On Smart homes and health Telematics (ICOST 2016), May 2016, Wuhan, China. pp.356-367. hal-01566918

\section{HAL Id: hal-01566918 https://hal.science/hal-01566918}

Submitted on 21 Jul 2017

HAL is a multi-disciplinary open access archive for the deposit and dissemination of scientific research documents, whether they are published or not. The documents may come from teaching and research institutions in France or abroad, or from public or private research centers.
L'archive ouverte pluridisciplinaire HAL, est destinée au dépôt et à la diffusion de documents scientifiques de niveau recherche, publiés ou non, émanant des établissements d'enseignement et de recherche français ou étrangers, des laboratoires publics ou privés. 


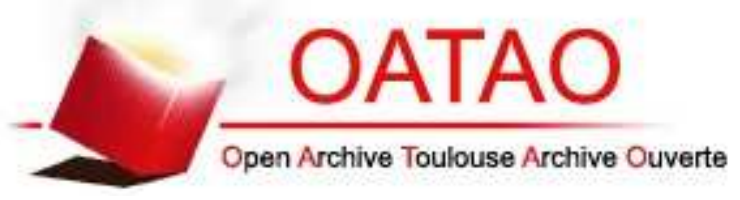

\section{Open Archive TOULOUSE Archive Ouverte (OATAO)}

OATAO is an open access repository that collects the work of Toulouse researchers and makes it freely available over the web where possible.

This is an author-deposited version published in : http://oatao.univ-toulouse.fr/ Eprints ID : 16947

The contribution was presented at ICOST 2016 :

http://www.icostconference.org/

To cite this version : Bougeois, Elizabeth and Duchier, Jenny and Vella, Frédéric and Blanc Machado, Mathilde and Van den Bossche, Adrien and Val, Thierry and Brulin, Damien and Vigouroux, Nadine and Campo, Eric Post-test perceptions of digital tools by the elderly in an ambient environment. (2016) In: 15th International Conference On Smart homes and health Telematics (ICOST 2016), 25 May 2016 - 27 May 2016 (Wuhan, China).

Any correspondence concerning this service should be sent to the repository administrator: staff-oatao@listes-diff.inp-toulouse.fr 


\title{
Post-test perceptions of digital tools by the elderly in an ambient environment
}

\author{
Elizabeth Bougeois ${ }^{4}$, Jenny Duchier ${ }^{3}$, Frédéric Vella ${ }^{1}$, Mathilde Blanc Machado ${ }^{1}$, Adrien \\ Van den Bossche ${ }^{1}$, Thierry Val ${ }^{1}$, Damien Brulin ${ }^{2}$, Nadine Vigouroux ${ }^{1}$, Eric Campo ${ }^{2}$ \\ ${ }^{1}$ IRIT, Université de Toulouse, CNRS, INPT, UPS, UT1, UT2J, Toulouse, France \\ ${ }^{2}$ LAAS-CNRS, Université de Toulouse, UT2J, Toulouse, France \\ ${ }^{3}$ INSERM, UMR 1027 INSERM-UPS, Toulouse, France \\ ${ }^{4}$ LERASS, UT2J, Toulouse, France \\ \{Mathilde.Blanc-Machado,vella,vigourou\}@irit.fr, \\ \{vandenbo,thierry.val,elizabeth.bougeois,eric.campo\}@univ-tlse2.fr \\ jenny.duchier@inserm.fr
}

\begin{abstract}
This article presents the first results about the perception of 33 elderly people about interaction tools in a digital ambient environment. The evaluation of this study is based on the filling of a short questionnaire, and interviews after a presentation of the different possible technologies and the use of a digital automated living lab, centred on a life scenario, by volunteers. The first results show a significant interest of the elderly in the voice interaction mode, in comparison with tactile interaction or switches, as well as a good opinion of the technological tools presented, in the context of a potential future use, for their health, better autonomy and well-being.
\end{abstract}

Keywords: Ambient digital environment, usability, perception, interaction mode, elderly, Living Lab.

\section{Introduction}

Longer life increases the number of people with loss of autonomy and may lead to dependence [1]. Needs in terms of securing a dependent elderly person, or a disabled person and needs for a response to isolation and social ties deficit have been identified as well as key ingredients [2] and challenges [3] for Ambient Assisted Living (AAL) systems [4]. The issue of home support is justified by a social necessity and by individual aspirations or for savings in health spending and social protection [5].

The use of ICT (Information Technology and Communication) can enable frail people or people with disabilities to live better by giving them the means to be more independent at home [6]. Many efforts have been made to increase accessibility to ICT -communication devices, assistive and health technologies ...- for old people but the solutions are not yet at the appointment. This is mainly due to the lack of study of the real needs and a clear definition of the elderly profiles (dependent, frail, socially isolated, digital exclusion, etc.). Some specific characteristics prevent or put a brake on the access to these new technologies. The behaviour and interaction modes of these people in their use and access to these technologies as well as their perception of technology are not fully understood because of the many influencing factors. 
$[7,8]$ explored the potential for voice user interface to interact with home-based services. [9] reported studies comparing touch screen and traditional mouse input and [10] described the design and the use of handheld computers for older adults. These modalities of interaction adapted to the home are still a challenge in connection with home automation and ambient technologies. Another significant challenge when studying technologies within the True Life Lab concept [11] is the living context where the research is carried out to inform about the acceptability of future innovative technologies or services.

Methods and tools from social sciences and ICT need to be merged to explore and analyse the behaviour of users with these innovative technologies to design, adapt them, on the basis of related ICT experiences. It is also important to consider how a new numeric ambient technology including intuitive interaction based on gesture, tactile and speech enables to fit the profile of the user.

Therefore, a methodology [12] mixing ethnographic observation [13] of actions and interviews [14] needs to be investigated. This innovating methodology must take into account several restraints, for instance the fact that the elderly people cannot endure a long experimentation time and may be stressed by the numeric ambient environment with observers.

We propose an interdisciplinary methodology to anticipate the use and the acceptance of these technologies by an elderly population, to build real models of habitat and solutions tailored to their expectations. In this way, it is necessary to study the relationship of these people with digital technologies in a smart home environment. This is the reason why the paper presents the preliminary results of an experiment conducted in living lab on a sample of 33 volunteers (aged $>60$ years). A test protocol was implemented, so that the volunteers could test the technological tools available in a smart home and-perform, alone, independently a real-life test scenario. The last step of the study was the filling of a questionnaire on the utility and relevance of the tools used. The change in perception about the technologies used between the before and after testing was particularly studied.

The paper is organized as follows: Section 2 describes the experimental protocol. Section 3 presents the test environment. Section 4 gives the method and the observation tools. Section 5 gives preliminary results. Section 6 offers a discussion. Section 7 ends the paper with a conclusion and comments about future work.

\section{Experimental protocol}

The only criteria retained for the inclusion of participants in this project are the age at least 60 years of age- and being retired. There is no exclusion criterion about diseases, disabilities or loss of independence.

Up to three participants are called on a half day during which researchers accompany them. The experiment session is divided into seven phases:

- The signature of a consent letter with the presentation of the scientists' team, the objectives of the study and the different phases of the experiment.

- The response to a questionnaire on a tactile tablet. A researcher accompanies each participant to help him to use the tablet or to understand the questions. The goal of 
this questionnaire is to know socio-cultural profiles of elderly, their current uses of ICT, the intentions of using new technologies, the anticipation of the place of settlement and their adaptation.

- The visit of a smart home (cf. section 3) and the presentation of the different technologies and services available. Participants discover the smart home where they will carry out a scenario and they can ask any questions concerning the functioning of the equipment.

- The appropriation period of the three different interaction techniques. This phase allows participants to try classic switches and the touch screen user interface to control the home equipment. They are also informed that they can speak with the smart home but without spoken example. This choice was retained to avoid influencing the participant and to let them discover this interaction mode during the test.

- The individual experimentation into the smart home, according to a scenario simulating the awakening of the participants. They are asked to carry out their usual activities after getting up in the morning. They can use classic switches and/or tactile interface on tablets and/or voice control of the smart home.

- The post-experimentation semi-structured interview with researchers in human and social sciences. The researchers have observed the participants during the scenario. By mixing the information collected both during the observation phase and during the interviews, the researchers aim to understand the participant's behavior, to discuss about their feelings and their projection in the use of the ambient technologies.

- The response to a post-experimentation questionnaire. This step enables to quantify the qualitative data from the previous interview.

\section{Smart home test environment}

The experimentation was set up in the smart home of the university institute of technology of Blagnac in France (MIB), [15]. This smart home is a technological, scientific and human platform. In the standard configuration, this flat of $80 \mathrm{~m}^{2}$ is equipped with a networking infrastructure adaptable to a valid, a frail or a disabled person (Fig. 1). It enables interconnectivity between commercial equipments and devices for intelligent management of the living environment, the implementation of technological solutions for monitoring and study of uses in the home setting. This home is equipped with various kinds of sensors (comfort, domestic security, access management, remote assistance...) and technical aids. The person can use classic switches, a touch screen interface (Fig. 2), or his/her voice. Interoperability is an important issue because of the heterogeneity of technological devices [16]; Interoperability is ensured through a middleware accepting standard protocols such as KNX or HTTP and also proprietary wireless protocols.

4 functions are proposed:

- E-health functions to follow the health-status of the person,

- Comfort and assistive functions to support person with automatism equipment,

- Access functions for safety and security, 
- Communication functions to control home equipment and to keep social ties with the outside (family, caregivers...).

In this study, only comfort and assistive functions are considered (Fig. 2).

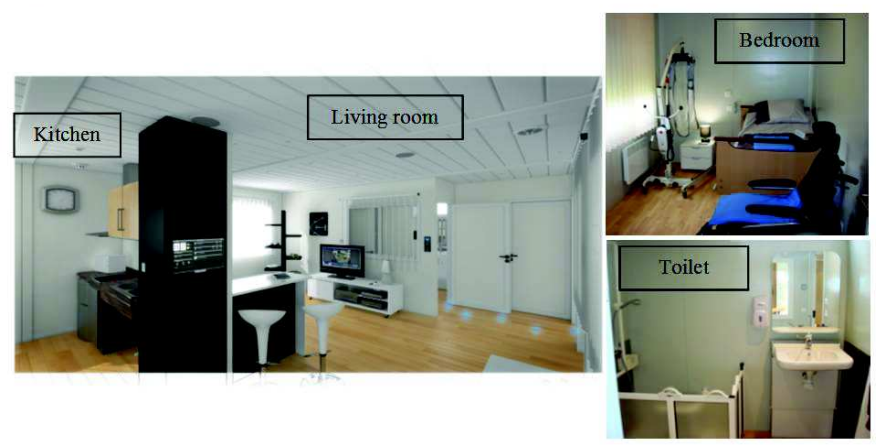

Fig. 1. The experimental smart home [15]

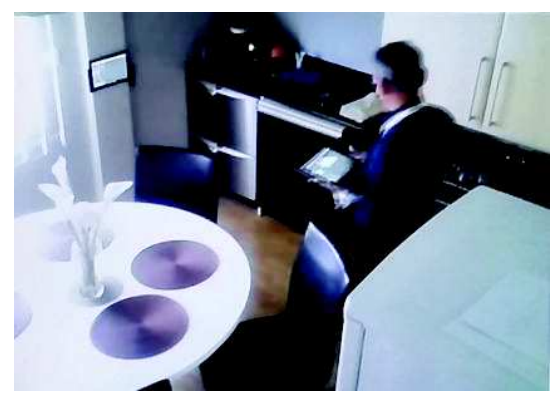

Fig. 2. A volunteer using the tablet in the kitchen

The control switches are completed with pictograms (kind of help) to explain the role of each button (Fig. 3-a). The tablet has an intuitive user interface with buttons affected to each room. Ergonomic criteria -size of buttons, space between buttons [17], and background colour to maximize the contrast- have been applied to facilitate the accessibility by older persons. Pressing the affected button provides access to the list of possible equipment commands. Same pictograms are used for the buttons on tablet and wall switches (Fig. 3-b). Voice input is recorded through an ambient sensitive microphone set up in the flat. The spoken message is displayed in the smart home by means of a text-to-speech synthesis.

A platform (called MIOZ) was designed to simulate the process chain of a spoken dialogue -speech recognition, understanding system, management of dialogue and generation of message- between the participant and the smart home without the knowledge of the participant. MIOZ sends and receives orders and feedbacks to sensors, actuators... regardless of the communication technology available in the MIB. Thus, the experimenter can track the participant's actions through the sensors' feedback and interact with him through. To achieve this goal, a specific lightweight middleware "MiCom" has been developed. MiCom enables connections with various 
technologies and insure interoperability thanks to a HTTP-based command set to interact with sensors and actuators, regardless of the communication technology. Each device of the environment can be managed by calling HTTP URLs.

The participant is also observed thanks to four cameras deployed in the smart home.
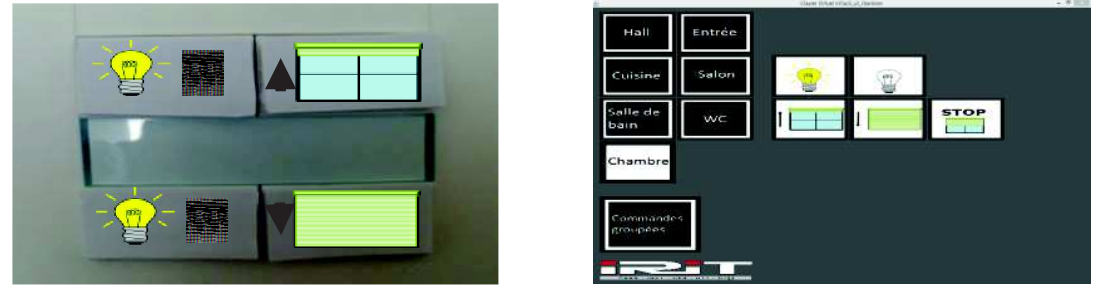

Fig. 3. a- Pictograms associated to wall switches b- Touch screen user-interface

\section{Method and observation tools}

\subsection{Ethnography and communication tool}

The social science process of the study is inductive, based on grounded theories. The analysis of the qualitative data is focused both on the actions performed by the elderly people and their behaviour during the observation stage, and on their discourse about their experiment, during the interviews.

The interview lasts between 15 and 20 minutes and is focussed on five items:

- How the participant felt during the experiment?

- Which ICT the participant likes best and which are convincing?

- What could be the participant's motivation to use the ICT at home?

- When? -that is to say: anticipation of the adaptation of the home and intentions in terms of investment,

- In case of social contribution: which ICT would come first and how is the participant ready to carry out administrative procedures?

Following the interview, the short questionnaire is presented on tablet. This questionnaire is presented as a resume of the total experiment. It focusses on the opinion of the participant about ICT's.

\subsection{Questionnaire}

According to the experiment protocol (cf. section 2) participants were invited to answer to a questionnaire focussed on four items:

- The view about technologies,

- The wish to settle domotic equipment and ICT at home. If positive answer: which items? (Voice input system, talking machine, touch screen user interface, control switches, light path, carephone and adjustability of furniture to abilities and needs, 
several items could be selected). If negative answer: justification (no perception of usefulness, too expensive, lack of confidence in technologies, intrusive technology, fears about the use of collected data),

- Interaction mode preferences,

- Easiness of the different interaction modes (prioritisation).

The analysis of the whole data gives comprehensive elements of the way the elderly consider the use of ambient technologies dedicated to palliate the loss of autonomy.

\subsection{Justification of our approach}

Carrying out semi structured interviews enables the researchers to collect information that were not possible to anticipate: the participants engage, during the interviews, their authentic living experience as elderly people, their representations and projections about aging, loss of autonomy or dependence, their eventual fears about the future, their level of information and their representations about ICT's in an ambient environment.

Their discourse is therefore incarnated, grounded on their life experience and freely contextualized.

In such a protocol, this way of investigating enables to qualify quantitative data and gives a vision or orientation from which it is possible to define new tracks for the analysis of the results. This come and go between qualitative and quantitative information leads to precise, sharp and authentic consideration of human beings as far as their health is concerned and benefits into better understanding of their real needs. The qualitative investigation enriches the quantitative data, and vice versa.

\section{Results}

We have proceeded to an experimentation period of 33 volunteers and have carried out an analysis of both post experiment interviews and questionnaires.

\subsection{Population}

33 volunteers ( 21 female and 12 male) were recruited (Table 1). Among them, there is a person with motor impairment (lower limbs) and another with cognitive impairment.

Table 1 Population profile

\begin{tabular}{lcccc}
\hline & $60-64$ years & $65-74$ years & $75-90$ years and older & Total \\
\hline Female & 4 & 10 & 7 & 21 \\
Male & 2 & 8 & 2 & 12 \\
Total & 6 & 18 & 9 & 33 \\
\hline
\end{tabular}


All the others have no deficiency except visually impairment corrected by wearing glasses. All of them have signed the consent letter and agree with the goal of the experiment protocol.

\subsection{Quantitative results}

Fig. 4 shows that $90.91 \%$ of older people surveyed have changed their opinion on new technologies (about $87,88 \%$ in a positive way towards 3 in a negative way). $9.09 \%$ have not changed their opinion after the experiment.

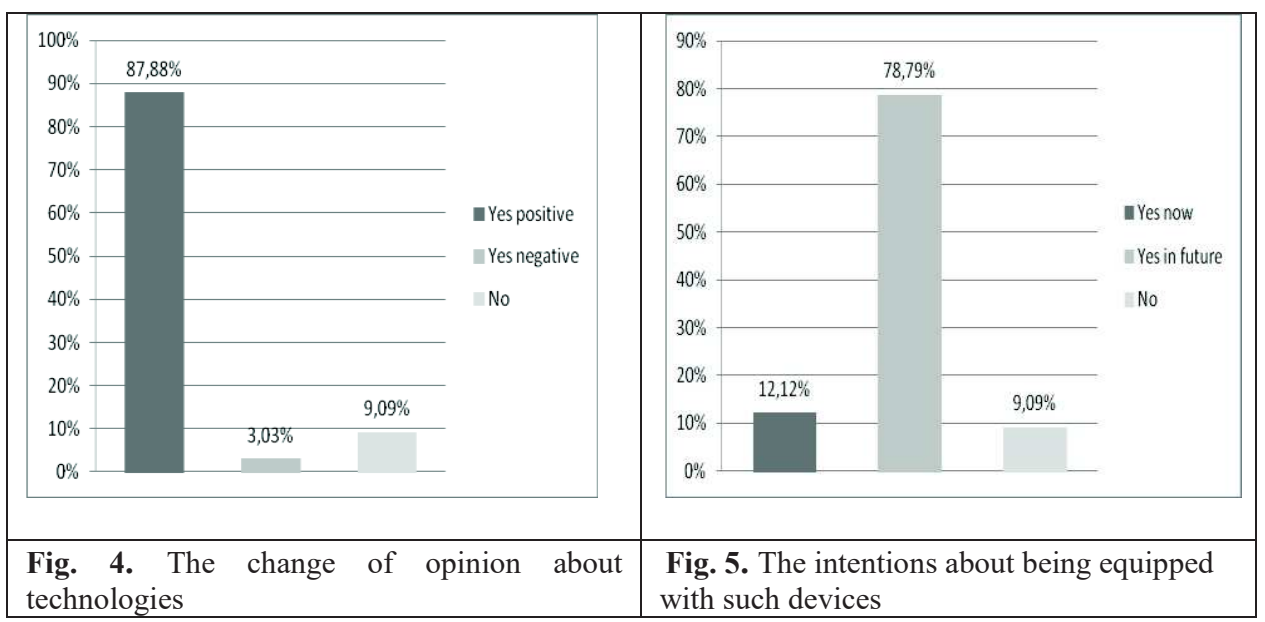

We notice that only $12 \%$ of elderly are ready to use currently the home automation and ICT technologies. However, when they are projected in the future, about $79 \%$ are interested in these technologies (Fig. 5). $81.82 \%$ of the participants say they are ready to carry out administrative procedures to adapt their home.

Fig. 6 shows the different wishes about ICT in smart home, several answers could be given by the participants for a same question. We can see a strong preference for speech technologies $(75.76 \%$ respectively for voice recognition and $57.58 \%$ for voice synthesis). The light path when the person gets up is also highly chosen $(51.52 \%)$. A lower interest $(42.42 \%)$ is reported for the touch screen user interface even if this interaction mode is available everywhere on tablet and smartphone. The lowest interests are respectively for control switches and carephone. These intention rates are surprising because of the usual use of control switches in a standard home and the importance of teleassistance for frail or dependent people. Negative reasons reported concerns mainly the cost $(6.6 \%)$ and rate equality $(3.3 \%)$, the lack of perception of usefulness and the potentially intrusive dimension. 


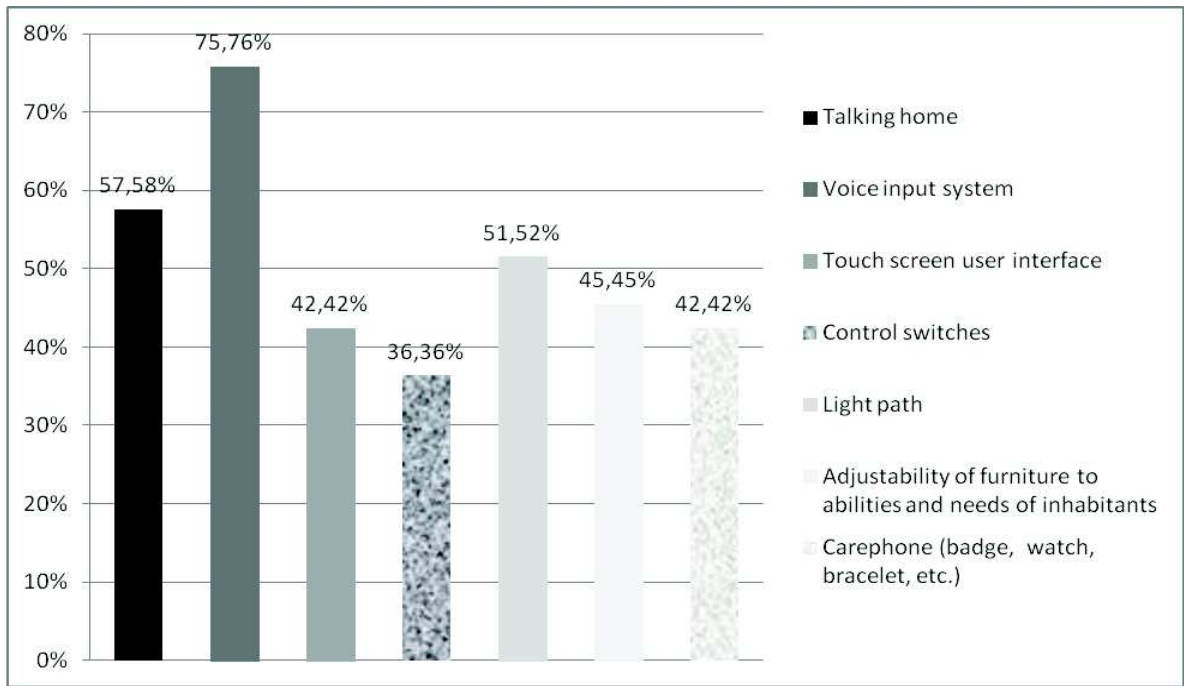

Fig. 6. Which devices at home?

Fig. 7 shows the high preference for voice input as interaction mode to control the smart home (about $70 \%$ as first choice). The tactile interaction appears as a second choice (about 55\%, Fig. 8) while the switch control is chosen as a third choice (about $58 \%)$. A Pearson's chi-squared test $(\chi 2)$ [18] was applied between the variables (interaction technique and range of selection). Pearson's chi-squared test data are: Table X-squared $=42, \mathrm{df}=4, \mathrm{p}$-value $=1.668 \mathrm{e}-08$. The interaction technique and the range of selection are dependant for the preference criteria. This means that there is a significant statistic link between these two variables.

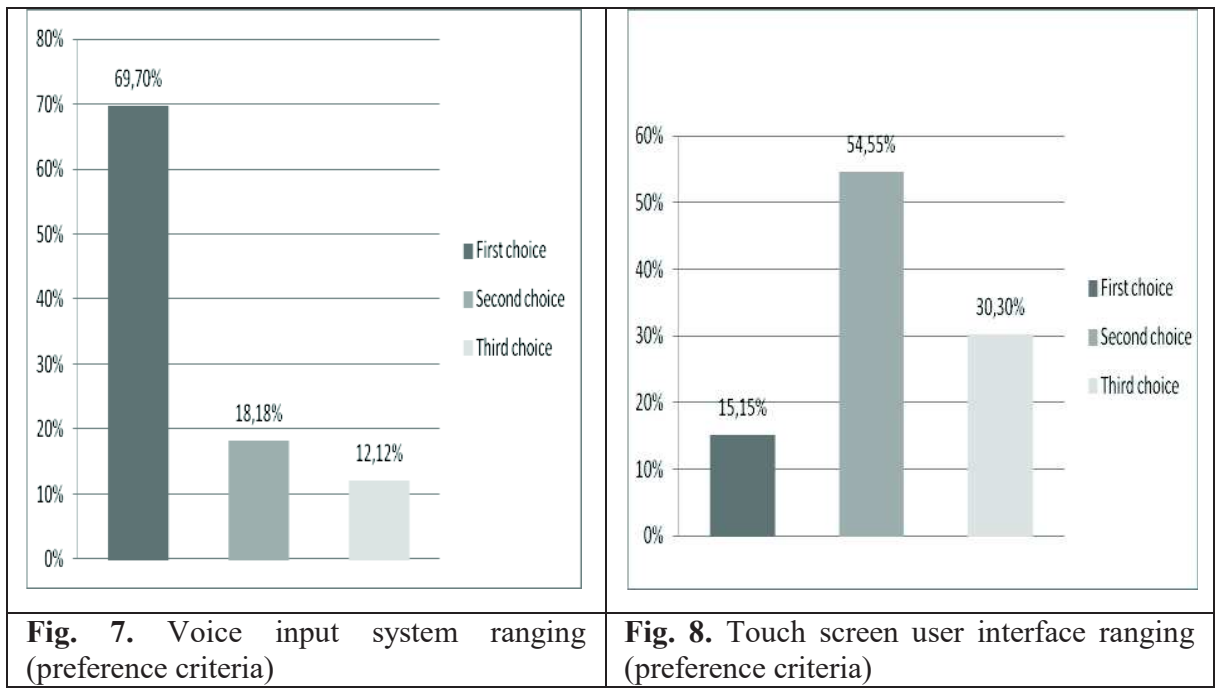


The same tendency is observed from the ease criteria (first choice for voice input (Fig. 9), second choice for touch screen interface (Fig. 10). However the rate for the second choice is respectively $(51.52 \%$ for touch screen and around $40 \%$ for switch control).

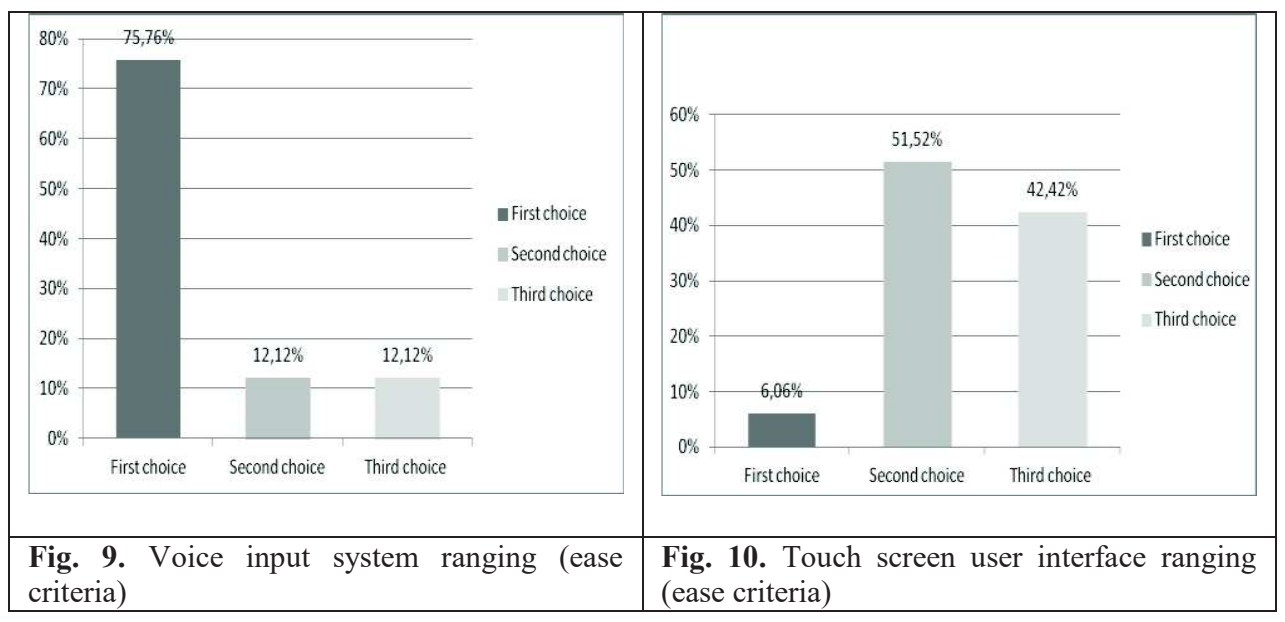

A Pearson's chi-squared test $(\chi 2)$ was applied between the variables (interaction technique and range of selection) for the ease criteria (Fig. 9 and Fig. 10). Pearson's chi-squared test data are: Table X-squared $=37.091, \mathrm{df}=4, \mathrm{p}$-value $=1.725 \mathrm{e}-07$. The two variables (interaction technique and range of selection) are also dependant from the ease criteria.

\subsection{Qualitative results}

First, the possible voice interaction with the MIB smart home through the MIOZ platform appears as the favorite equipment, from a qualitative point of view. It is perceived as a personalization of the smart home, a reassuring presence and a strong element of comfort: "the voice tranquilizes me, it reassures me"; "I am most impressed, amazed by the voice"; "one feels less lonely with the voice". Even when a negative perception occurs, it is nuanced: "the voice is stressful...but I suppose one gets used to it". This element matches with the quantitative results (cf. Fig. 6).

However, the most important result of the study is clearly the need to offer several modes of interaction to the inhabitant. The experimented protocol allows redundancy of the equipment, which gives, according to the discourse of the participants, the possibility of an extended comfort and well-being. This redundancy is perceived as a means of keeping acting and choosing the way orders are given. It is also perceived as a warranty of reliability of the different technologies as well as a possibility of doing several tasks or giving several orders simultaneously. It is viewed as non-restrictive as far as acting freely is concerned.

The participants sometimes had a defiant attitude towards ICT's before the experiment. This perception changed after. They express the fact that they had not 
expected such easiness in the use of the equipments: "I feared to be overwhelmed; these technologies now seem more feasible".

The cost of such equipments remains a problem, as the participants, even if they do not mention any amount; think that it is too expensive. It seems important to specify that, without any question about this aspect, they have spontaneously mentioned whether they were owners or tenants of their home. This particular point clearly orientated their thoughts and projections into the question of, on the one side, investment and, on the other side, equipment. Tenants tend to anticipate their future settlement in a nursing home.

\section{Discussion}

The technologies tested during the experiment appear to elderly people as a means of independence and a way to avoid going to a nursing home and stay healthy at home. However, some participants found that these innovative devices were too "medicalized", others wonder about the system reliability or costs at home. The novel interaction techniques (voice and tactile) are preferred: the voice input is natural and touch screen is fun and easy to use.

Even though the participants declare themselves as "concerned" and "convinced" by the technologies in the MIB, they also say that they don't need it immediately but in the future. Some of them clearly express their difficulty to project themselves into the loss of autonomy. This may be analyzed as ambivalence, or reluctance about palliative technologies as far as free and independent aging is concerned.

During the interviews, some participants have a contradictory discourse, compared to their behaviour during the experiment, such as, for instance, being very keen about the voice interaction with the MIB and not using it at all. It seems that the experience has changed their views positively as far as technologies are concerned. This remark has to be understood in the restraint context of the study: the experiment is new, enjoyable, both experiment and interviews were carried out in a short duration.

The corpus of participants up till now studied is very heterogeneous: age, social and cultural background, previous uses of technologies, representations and experience of aging...

The age of the participants (60 up to 90 years) does not seem to have any influence on the results: there is -in the ongoing results- no specificity in the use of the ICT's according to the age. These criteria are to be taken into account because the ongoing results do not always reflect this heterogeneity: the further experiments will allow a more accurate analysis.

\section{Conclusion}

This paper proposes a new methodological approach for a better understanding of the needs of the elderly and the use of technologies in an ambient digital environment -in 
a living lab- based on the implementation of a multidimensional observation tool. This method interconnects a qualitative regard with quantitative data.

The ongoing results lead to a new question, related to the stability of the preferences, in terms of ICT modalities, in the long term.

This remark, linked with the heterogeneous characteristics of the elderly, enables to anticipate a further difficulty to qualify different profiles of users or ideal-types of inhabitants or modes of connected settlements.

The study aims a better understanding of the potential uses of ambient technologies by the elderly. The next step is to carry on the experiments with about one hundred more people, in order to sharpen the previous observations and to interpret these results according to the social, cultural and health profiles of the elderly.

The multimodal way of using ICT's appears as essential for a complete consideration of the different representations, needs, uses of the elderly, as well as for an optimized acceptability and accessibility of these technologies. Also, other interaction modes could be tested, such as gesture or partial body movement, which are more natural interactions.

Acknowledgments. This work is partially funded by the MSH-T of Toulouse and AG2R La Mondiale Insurance group.

\section{References}

1. Chappell N.L., Cooke H.A. 2010. Age Related Disabilities - Aging and Quality of Life. In: JH Stone, M Blouin, editors. International Encyclopedia of Rehabilitation. Available online: http://cirrie.buffalo.edu/encyclopedia/en/article/189/

2. Sun, H., De Florio, V., Gui N. and Blondia C. The missing Ones: Key Ingredients Towards Effective Ambient Assisted Living Sytems, Journal of Ambient Intelligence and Smart Environments archive. 2 (2) : pp. 109-120, (2010)

3. Coughlan, T., Mackley, K. L., Brown, M., Martindale, S., Schlögl, S. Mallaband, B. et al. Current Issues and Future Directions in Methods for Studying Technology in the Home. PsychNology Journal; 11(2): 159-184 (2013)

4. Bierhoff, I., van Berlo, A. Abascal, J. et al. Chapter 3. Smart home environment. Towards an inclusive future: Impact and wider potential of information and communication technologies. In: Roe P.R.W. (ed.): pp.110-156, (2007)

5. Coughlin, J., D'Ambrosio, L., Reimer, B., \& Pratt, M., Older adult perceptions of smart home technologies: implications for research, policy \& market innovations in healthcare. Engineering in Medicine and Biology Society, 2007. EMBS 2007. 29th Annual International Conference of the IEEE, pp. 1810-1815 (2007).

6. Curry, R., Trejo-Tinoco M., Wardle, D. The Use of Information and Communication Technology to Support Independent Living for Older and Disabled People. London: Department of Health (2003)

7. Schlögl, S., Chollet, G., Garschall, Tscheligi, M.M., Legouverneur, G. Exploring Voice User Interfaces for Seniors, 6th Conf. on Pervasive Technologies Related To Assistive Environments, Rhodes Greece, (2013)

8. Vacher, M., Portet, F., Rossato, S., Aman, F. Golanski, F., Dugheanu, R., Speech-based interaction in an AAL-context, Gerontechnology; 11(2): pp. 310-316, (2012) 
9. Findlater, L., Froehlich, J.; Fattal, K., Wobbrock, J. O.; Dastyar, T.: Age - Related Differences in Performance with Touchscreens Compared to Traditional Mouse Input. In: CHI'13, 2013 - ISBN 9781450318990, S. 1-4 (2013)

10. Zhou, J., Rau, P.-L. P., Salvendy, G., Use and Design of Handheld Computers for Older Adults: A Review and Appraisal. In: International Journal of Human-Computer Interaction Bd. 28, Nr. 12, pp.799-826 (2012)

11. Vigouroux, N., Rumeau, P., Boudet, B., Vella, F. and Salvodelli, M., Wellfar-e-link®: true life lab testing of a homecare communication tool. In: Non-pharmacological Therapies in Dementia, Nova Science Publishers; 3(2): pp.133-142, (2015)

12. Olivier de Sardan J.-P., Epistemology, Fieldwork, and Anthropology. Palgrave MacMillan (2015)

13. Goffman, E., The Interaction Order, American Sociological Review.48: pp.1-17, (1983)

14. Corbin, J. and Strauss A.L. Basics of Qualitative Research : Techniques and Procedures for developing Grounded Theory (4th ed) San Jose State University, Sage (2015)

15. Campo, E., X. Daran X. and L. Redon, L., Une maison intelligente au carrefour des sciences technologiques et des sciences humaines. 2nd Int. Conf. sur l'accessibilité et les systèmes de suppléance aux personnes en situation de handicap; Paris, France : pp.33-42, (2011)

16. Le Guilly, T.; Olsen, P.; Ravn, A.P.; Rosenkilde, J.B.; Skou, A., HomePort: Middleware for heterogeneous home automation networks, in Pervasive Computing and Communications Workshops (PERCOM Workshops), 2013 IEEE International Conference on , vol., no., pp.627-633, (2013)

17. Jin, Z. X., Plocher, T., and Kiff, L., Touch Screen User Interfaces for Older Adults: Button Size and Spacing. In Universal Access in Human Computer Interaction. Coping with Diversity, Vol. 4554, pp. 933-941, (2007)

18. Pearson, K., On the criterion that a given system of deviations from the probable in the case of a correlated system of variables is such that it can be reasonably supposed to have arisen from random sampling, Philosophical Magazine Series 550 (302): 157-175 (1990) 